\title{
Mycobacterium chelonae cutaneous infection in a patient with mixed connective tissue disease
}

\author{
Renan Lage ${ }^{1}$ \\ Felipe Borba Calixto Santos ${ }^{1}$ \\ Elisangela Samartin Pegas Pereira ${ }^{1}$
}

\author{
Danilo Guerreiro Zeolo Biccigo ${ }^{1}$ \\ Erica Chimara ${ }^{2}$ \\ Adilson da Costa ${ }^{1}$
}

\begin{abstract}
Around 50 mycobacteria species cause human disease. Immunosuppressive states predispose to nontuberculous mycobaterium infection, such as Mycobacterium chelonae: AFB, non-tuberculous, fast growth of low virulence and uncommon as a human pathogen. It may compromise the skin and soft tissues, lungs, lymph nodes and there is also a disseminated presentation. The diagnosis involves AFB identification and culture on Agar and Lowenstein-Jensen medium base. A 41-year-old female with MCTD (LES predominance) is reported, presenting painless nodules in the right forearm. She denied local trauma. Immunosuppressed with prednisone and cyclophosphamide for 24 months. Lesion biopsy has demonstrated positive bacilloscopy (Ziehl-Neelsen stain) and M.chelonae in culture (Lowenstein-Jensen medium base), therefore clarithromycin treatment has been started (best therapy choice in the literature).
\end{abstract}

Keywords: Clarithromycin; Immunosuppression; Mixed connective tissue disease; Mycobacterium chelonae; Mycobacterium fortuitum; Nontuberculous mycobacteria; Prednisone; Skin; Virulence

\section{INTRODUCTION}

Until the 1950s no non-tuberculous mycobacterium species had been identified as causing infection in human beings; however, at the current time, around 50 potentially pathogenic mycobacterium species have been isolated in humans.

Immunocompromised states, such as AIDS and immunosuppressive drug use, can facilitate nontuberculous mycobacterium infection, among which Mycobacterium chelonae, which is a fast-growing mycobacterium, involving mainly the lungs, but that has also been described as affecting the skin, lymph nodes and the skeletal system. ${ }^{1}$

\section{CASE REPORT}

A 41-year-old female patient was diagnosed with mixed connective tissue disease, of which the main component was systemic lupus erythematous with associated nephritis. Progressive onset of grow- ing painless lesions in her right forearm were described, for a three-month period, without any other symptoms, previous trauma, local surgery or injections. She had been using prednisone one $\mathrm{mg} / \mathrm{kg} /$ day (immunosuppressive dose) and regular cyclophosphamide pulses for 24 months.

The physical examination showed erythematous nodules, discreetly hard, palpable be rather than visible, with a diameter of one and a half to two centimeters, distributed along the anterior and posterior sides of the right forearm (Figure 1).

She underwent a skin lesion biopsy and the anatomopathological exam demonstrated: extensive adipocytic necrosis in the hypodermis with lipomembranous and pseudo cystic patterns, associated with recent hemorrhage and subtle inflammatory infiltrate surrounding some vessels, localized in superficial and deep dermis, sometimes in the hypodermis, com-

Received on 13.11.2012.

Approved by the Advisory Board and accepted for publication on 21.10.2013.

* Work performed at the Faculdade de Medicina Departamento de Dermatologia - Pontifícia Universidade Católica de Campinas (PUC-Campinas) - Campinas (SP), Brazil.

Financial Support: none

Conflict of Interests: none

Pontifícia Universidade Católica de Campinas (PUC Campinas) - Campinas (SP), Brazil.

Instituto Adolfo Lutz - São Paulo (SP), Brazil. 


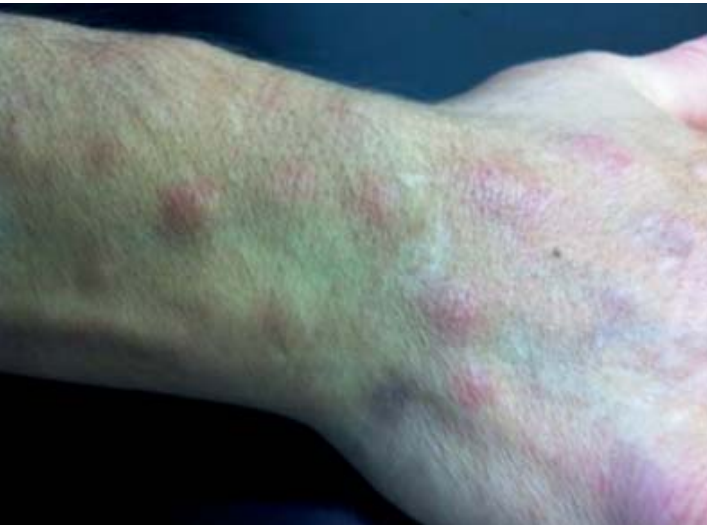

FIGURE 1: Erythematous nodules, discreetly hard, with a diameter of $1.5-2.0 \mathrm{~cm}$, distributed along the anterior and posterior sides of the right forearm



FIGURE 2: Extensive adipocytic necrosis in the hypodermis with lipomembranous and pseudocystic patterns, associated with recent hemorrhage and subtle inflammatory infiltrate surrounding some vessels, localized in superficial and deep dermis, sometimes in the hypodermis, composed of lymphocytes, histiocytes and plasmacytes



FIGURE 3: Extensive adipocytic necrosis in the hypodermis with lipomembranous and pseudocystic patterns, associated with recent hemorrhage and subtle inflammatory infiltrate surrounding some vessels, localized in superficial and deep dermis, sometimes in the hypodermis, composed of lymphocytes, histiocytes and plasmacytes M.fortuitum complex, acid-fast bacilli, non-pigmented and with a fast rate of growth; it was first isolated from a sea turtle named Chelona corticata, but can be found in the water, soil, dust and contaminated instruments. ${ }^{2}$

Although these mycobacteria have the remarkable characteristic of producing biofilm, they have low virulence, and therefore do not usually provoke disease in humans. However, immunosuppressive status (transplant recipients; those with AIDS; immunosuppressive drug use, and long term corticotherapy) have led to infections, caused by these organisms in humans since the 1950s. ${ }^{3,4}$ It has also been recognized that this kind of infection may appear in non-immunocompromised people, but also in those with lung base disease or those who have undergone some invasive procedure, as the simple act of shaving or major operations, due to a residual break in the skin or potentially contaminated instruments. ${ }^{5}$

After the incidental environmental inoculation, the incubation period lasts from four to six weeks. ${ }^{2}$ The infection may involve: the skin, soft tissues, lungs, lymph nodes (mainly in small children), osteoarticular system, liver and also trigger a disseminated presentation. The degree of severity depends on the mycobacterium virulence, but chiefly on the immune status of the carrier; thus immunosuppressed people 


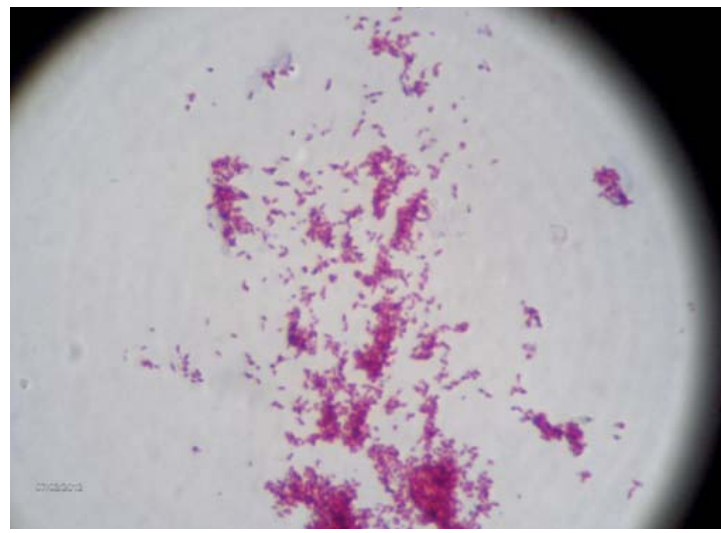

FIGURE 4: Ziehl-Neelsen coloration demonstrating AFB in cutaneous biopsy material

are more vulnerable to serious and disseminated presentations. ${ }^{2}$ Cutaneous manifestations include edema, papules, hard-to-heal ulcers, abscess, subcutaneous nodules and multiple or isolated chronic drainage fistulas; they usually appear in the extremities, mainly the lower ones. In the reported case multiple subcutaneous nodules were found in the right upper limb.

The diagnostic confirmation using affected tissue includes: bacilloscopy (identification of the acidfast bacilli using Ziehl-Neelsen coloration), histopathological study (demonstrates tuberculous or sarcoid granulomas, rheumatoid nodules, diffuse dermal and/or hypodermal histiocytic infiltration and chronic panniculitis), molecular biology methods (PCR is most commonly used, it is highly effective and can return a result in under two hours; however, it does not show the antibiotic susceptibility), culture in appropriate mediums (e.g. agar and Lowenstein-Jensen, which provide culture in about one week), essential for the therapeutic decision because mycobacteria are resistant to many common drugs, and finally RNA probes. ${ }^{1,6,7}$

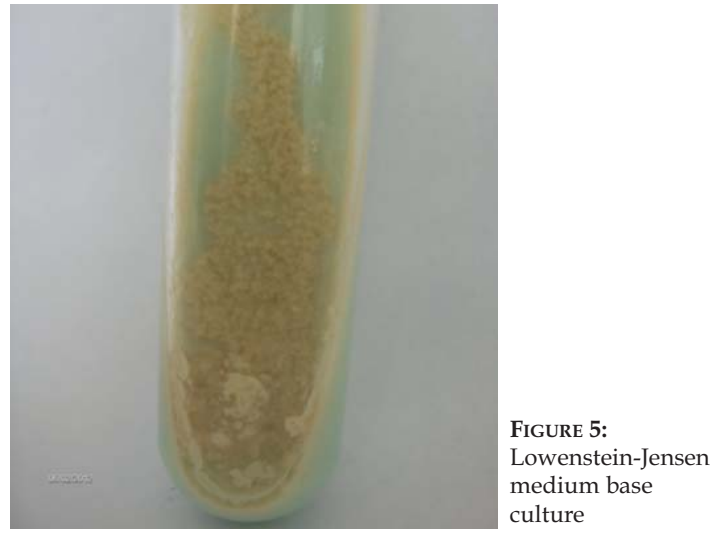

The recommended therapy differs from that used for Mycobacterium tuberculosis, because nontuberculous mycobacteria are generally resistant to antituberculous drugs and metronidazole, even though the latter has been approved by the FDA for treating this kind of infection. ${ }^{3}$ There are also reports of resistance with the use of cefotaxime, doxycycline, ciprofloxacin and sulfamethoxazole. ${ }^{8}$ There is no randomized control study demonstrating a specific therapy for these infections, nevertheless clarithromycin (without a stated dose, the most commonly used is $500 \mathrm{mg} /$ day) has been prescribed successfully, with few reports of resistance. ${ }^{1,9}$ The concomitant use of another drug, preferably ciprofloxacin or levofloxacin, has been widely employed in order to prevent resistance. ${ }^{1}$ Although the duration of therapy has not been defined, it should be around four to six months, or in another scheme it may be used from diagnosis until four to six weeks after the lesion has healed..$^{10}$ Surgical procedures, such as curettage and drainage, may also be performed. ${ }^{10}$ 


\section{REFERENCES}

1. Lee WJ, Kang SM, Sung H, Won CH, Chang SE, Lee MW, et al. Non-tuberculous mycobacterial infections of the skin: A retrospective study of 29 cases. J Dermatol. 2010;37:965-72.

2. Halpern J, Biswas A, Cadwgan A, Tan BB. Disseminated cutaneous Mycobacterium chelonae infection in an immunocompetent host. Clin Exp Dermatol. 2010;35:269-71.

3. Esteban J, Ortiz-Pérez A. Current treatment of atypical mycobacteriosis. Expert Opin Pharmacother. 2009;10:2787-99.

4. Bamias G, Daikos GL, Siakavellas SI, Kaltsa G, Smilakou S, Katsogridakis I, et al. Atypical mycobacterial infection presenting as persistent skin lesion in a patient with ulcerative colitis. Case Rep Med. 2011;2011:480987.

5. Murray KN, Bauer J, Tallen A, Matthews JL, Westerfield M, Varga ZM. Characterization and management of asymptomatic Mycobacterium infections at the Zebrafish International Resource Center. J Am Assoc Lab Anim Sci. 2011;50:675-9.

6. Guarin N, Budvytiene I, Ghafghaichi L, Banaei N. Comparison of real-time polymerase chain reaction and conventional biochemical methods for identification of Mycobacterium chelonae-Mycobacterium abscessus group to the species level. Diagn Microbiol Infect Dis. 2010;67:333-6.

7. Hay RJ. Mycobacterium chelonae - a growing problem in soft tissue infection. Curr Opin Infect Dis. 2009;22:99-101.
8. Monego F, Duarte RS, Nakatani SM, Araújo WN, Riediger IN, Brockelt S, et al. Molecular identification and typing of Mycobacterium massiliense isolated from postsurgical infections in Brazil. Braz J Infect Dis. 2011;15:436-41.

9. Mukherjee T, Boshoff H, Barry CE 3rd. Comment on: Identification of antimicrobial activity among FDA-approved drugs for combating Mycobacterium abscessus and Mycobacterium chelonae. J Antimicrob Chemother. 2012;67:252-3.

10. Schneider P, Monsel G, Veziris N, Roujeau JC, Bricaire F, Caumes E. Successful treatment of nodular lymphangitis due to Mycobacterium chelonae in two immunosuppressed patients. Dermatol Online J. 2011;17:8

\author{
MAILING ADDRESS: \\ Renan Lage \\ Av. John Boyd Dunlop, $s / n^{\circ}$ \\ Jardim Ipaussurama \\ 13060-904 Campinas - SP \\ Tel: (19) 33438496 \\ E-mail: renanmedxxxv@yahoo.com.br
}

How to cite this article: Lage R, Biccigo DGZ, Santos FBC, Chimara E, Pereira ESP, Costa A. Mycobacterium chelonae cutaneous infection in a patient with mixed connective tissue disease. An Bras Dermatol. 2015;90(1):104-7. 